\title{
Virtual Thermal Sensor for Real-Time Monitoring of Electronic Packages in a Totally Enclosed System
}

This paper was downloaded from TechRxiv (https://www.techrxiv.org).

\section{LICENSE}

CC BY 4.0

SUBMISSION DATE / POSTED DATE

$12-11-2021 / 13-11-2021$

\section{CITATION}

Ahn, Chang-uk; Kim, Hwi-Su; Oh, Seungin; Kim, Jin-Gyun; Park, Dong II (2021): Virtual Thermal Sensor for Real-Time Monitoring of Electronic Packages in a Totally Enclosed System. TechRxiv. Preprint. https://doi.org/10.36227/techrxiv.16992907.v1

$\mathrm{DOI}$

10.36227/techrxiv.16992907.v1 


\title{
Virtual Thermal Sensor for Real-Time Monitoring of Electronic Packages in a Totally Enclosed System
}

\author{
Chang-uk Ahn, Student Membership, Seungin Oh, Student Membership, \\ Hwi-Su Kim, Membership, Dong II Park, Membership, and Jin-Gyun Kim, Membership
}

\begin{abstract}
A monitoring system is essential for controlling for the temperature under safe levels of operation. However, it is often challenging to attach temperature sensors directly to drive chips owing to the operating environment or geometric challenges. Based on this motivation, we present a virtual thermal sensing technique for the real-time temperature monitoring of the electronics package. A few real sensors located far from the target position are utilized in this virtual sensing system. These are then connected to a well-tuned finite element model for data augmentation utilizing an inverse heat conduction framework. Virtual sensors can estimate the temperature without the aid of a sensor installed inside but has a computational burden and stability issue. In order to address these challenges, a block diagonalization based on the Schur complement is employed to reduce the computational cost and employ a Tikhonov regularization for stability. Furthermore, an efficient ridge estimator is proposed to overcome the difficulty of selecting an optimal regularization parameter. The proposed ridge estimator could provide a reasonable initial estimation of the regularization parameter without collecting measurement data in advance. Finally, the proposed virtual thermal sensor was experimentally validated utilizing a sealed cylindrical structure in which the commercial servo drive operated.
\end{abstract}

Index Terms-Virtual thermal sensor, electronic packages, Tikhonov regularization, finite element method, inverse heat conduction problem, ridge estimator.

Manuscript received Month $\mathrm{xx}, 2 \mathrm{xxx}$; revised Month $\mathrm{xx}, \mathrm{xxxx}$; accepted Month $\mathrm{x}, \mathrm{xxxx}$. This research was funded by National Research Foundation of Korea (NRF-2018R1A1A1A05078730) and MOTIE, Korea Government (grant No. 20001228).

The first author works with the Department of Mechanical Engineering (Integrated Engineering), Kyung Hee University, 1732, Deogyeongdaero, Giheung-gu, Yongin-si, Gyeonggi-do 17104, Korea and with the Department of Robotics \& Mechatronics Research, Korea Institute of Machinery and Materials, Daejeon, 34103, Republic of Korea (e-mail: changuk.ahn@gmail.com).

The second author works with the Department of Mechanical Engineering (Integrated Engineering), Kyung Hee University, 1732, Deogyeong-daero, Giheung-gu, Yongin-si, Gyeonggi-do 17104, Korea (e-mail: seungin.oh@gmail.com).

The third author works with the Department of Robotics \& Mechatronics Research, Korea Institute of Machinery and Materials, Daejeon, 34103, Republic of Korea (e-mail: hskim81@kimm.re.kr).

The fourth author works with the Department of Robotics \& Mechatronics Research, Korea Institute of Machinery and Materials, Daejeon, 34103, Republic of Korea (corresponding author to provide e-mail: parkstar@kimm.re.kr).

The fifth author works with the Department of Mechanical Engineering (Integrated Engineering), Kyung Hee University, 1732, Deogyeongdaero, Giheung-gu, Yongin-si, Gyeonggi-do 17104, Korea (corresponding author to provide e-mail: jingyun.kim@khu.ac.kr).

\section{INTRODUCTION}

This study presents a virtual thermal sensor (VTS) for the real-time monitoring of electronic packages. Recently developed electronic packages are compact enough to fit in various limited spaces; thus, no control cabinet is required. Accordingly, drive modules integrated with the electronics package have been designed, leading to more critical thermal challenges; therefore, it is necessary to monitor the electronics package. However, it is challenging to attach temperature sensors directly (or appropriately located) to the drive chips because of the enclosed structure or operating environment. The VTS can be utilized to provide cost-effective alternatives to costly and/or impractical physical measurement instruments. The VTS solely requires a few temperature sensors installed at an easily accessible location in the machine to estimate the thermal quantity of interest. It can be applied in several applications, such as electric vehicle batteries, electric motors, semiconductor manufacturing thermal processors, transformers, etc. [1]-[4]. For a similar purpose, the virtual sensor technique has also been studied for structural vibration problems [5], [6].

The proposed VTS is based on the inverse heat conduction problem (IHCP) [7], [8]. Developing the VTS starts from modeling the heat transfer model. Updating thermal parameters and the filtering measurement errors are particularly added for accuracy and stability, respectively. It is also essential to determine the location and number of sensors. When estimating only one heat source, the closer the sensor is to the heat source, the better it is. Unconditionally increasing the number of sensors rather amplifies the measurement noise and should be avoided. When estimating multiple heat sources, it is desirable to select the location and number of sensors to have linearly-independent sensitivity coefficients with large magnitude [7], [8].

For heat transfer modeling, the finite element method (FEM) [9] is applied here. FEM provides highly accurate numerical results, but it is challenging to be employed for real-time monitoring because of its high computational burden. This can be alleviated by using the lumped thermal model [1], [3], [4], [10], but the lumped model may cause significant estimation errors in the IHCP. To address this challenge, a block diagonalization based on the Schur complement is employed. [11]. The consistency of the matrix can be condensed 
by the Schur complement, and the computational cost becomes much smaller than the original forms without significant loss of accuracy. This block diagonal matrix can be used in the real-time monitoring process with IHCP.

During the FE modeling process, the thermal model parameters of the numerical model are tuned to reflect the real model well. Test measurement data are used to revise the FE model. This task is commonly referred to as the model update [12], [13]. Most of this research has focused on two approaches in thermal problems: the steady-state method [14] and the transient method [15]-[18] . Steady-state methods commonly require long duration experimental runs and it is difficult to calibrate the specific heat capacity. Therefore, the transient approach based on the IHCP with a relatively brief duration experimental run is employed in this work to achieve the realtime monitoring. The conjugate gradient method based on the whole-time domain [8], [15], [16] was used to solve the IHCP. Three thermal model parameters, namely, thermal conductivity, specific heat capacity, and thermal contact conductance, are updated in the target electronic packages. It is difficult to adjust the three parameters simultaneously. Thus, at the component level, the thermal conductivity and the specific heat capacity are concurrently revised first, and the thermal contact conductance is adjusted in the assembly model.

In addition, measurement errors should be carefully controlled in VTS because small errors are dramatically amplified through the inverse problem. To address this issue, many researchers have developed the stabilizers such as the sequential function specification method (SFSM) [7], regularization method (RM) [19], [20], iterative regularization (IR) [21], and combined SFSM-RM [22]. These methods provide good stabilization performance, but those may require additional computational burden at every time step to reduce the illconditioning of the gain coefficient matrix. However, the Tikhonov regularization method [19], [20] only has a computational load when selecting the optimal Tikhonov parameter in pre-processing, not real-time sensing. Therefore, the Tikhonov method is considered in the proposed VTS.

The optimal Tikhonov parameter can be selected by the ridge estimator, such as the Morozov discrepancy principle [23], [24], generalized cross-validation (GCV) [25], [26], and L-curve rule [27], [28]. The most commonly used method is the Morozov discrepancy principle, which is relatively accurate and easy to use. This method can only be used when prior information is known [23], [24]. Currently, the noise level of temperature is well known; thus, the Morozov discrepancy principle is one of the best methods to recover the temperature history as accurately as possible, but it cannot guarantee the heat source estimation [29]. In addition, if the system size and data measurement time are increased, the conventional ridge estimator requires significant computational costs. This is because the discrepancy principle needs to calculate transient solutions at each regularization parameter discretized over a specified range. To improve this problem, we utilized an efficient ridge estimator proposed in the optimal hybrid parameter selection algorithm [30]. The key advantage of an efficient ridge estimator does not depend on FE transient solutions, unlike the conventional ridge estimator. Thus, regardless of the operating time of the system, the optimal Tikhonov parameter can be estimated quickly. This is possible because it is not based on the total square error but the sum of the bias and the variance errors. Each of the two errors is a function of the maximum change in heat flux and the noise level of the sensor, not transient solutions. In this study, a new ridge estimator for temperature estimation, rather than heat flux, is derived and used to maximize the performance of the real-time temperature sensing of the VTS.

Finally, the proposed VTS can be applied for the real-time monitoring of electrical machines with complicated heat flows and is accurate and stable. The methodology is demonstrated using a commercial servo drive, Gold Solo Twitter, which delivers up to $5 \mathrm{~kW}$. The commercial servo drive operates inside a sealed cylindrical housing. This paper is organized as follows. Section II reviews the thermal modeling and presents a solution algorithm that includes the block diagonal formulation based on the Schur complement. Section III introduces the model update. Section IV suggests the virtual sensing algorithm using the Tikhonov regularization method and the ridge estimator. Section $\mathrm{V}$ evaluates the feasibility and performance of the proposed VTS. Section VI presents the conclusions.

\section{THERMAL MODELING AND SOLUTION ALGORITHM}

This section briefly reviews the discretization model of the heat equation and the time integration scheme. We then presents a model order reduction technique based on the Schur complement [11] for real-time sensing. The governing equations for the temperature fields can be approximated by the discretization method as the following algebraic differential equation [9]:

$$
\mathbf{C} \dot{\mathbf{T}}(t)+\mathbf{K} \mathbf{T}(t)=\mathbf{q}(t),
$$

where $\mathbf{C}$ and $\mathbf{K}$ are the heat capacity and heat transfer matrices, respectively. $\mathbf{T}$ and $\mathbf{q}$ are the temperature and thermal loading vectors, respectively. If the total number of degrees of freedom (DOF) is defined as $N_{g}$, the matrix size of $\mathbf{C}$ and $\mathbf{K}$ is $\left(N_{g} \times N_{g}\right)$. The vector size of $\mathbf{T}$ and $\mathbf{q}$ is $\left(N_{g} \times 1\right)$. The relationship between the thermal parameters and the system matrices in Eq. (1) is presented in TABLE I.

To solve Eq. (1), the Backward Euler method is employed, which is given by

$$
\mathbf{T}_{m}=\mathbf{T}_{m-1}+\Delta t \dot{\mathbf{T}}_{m}
$$

where subscript $m$ denotes a time step, and time $t$ is represented as the discretized form $t_{m} . \Delta t$ denotes the time step size (or measurement time interval) and is defined as $\left(\Delta t=t_{m}-t_{m-1}\right)$. Then, we obtain

$$
\begin{gathered}
\mathbf{T}_{m}=\mathbf{A}^{-1}\left(\frac{1}{\Delta t} \mathbf{C} \mathbf{T}_{m-1}+\mathbf{q}_{m}\right) \\
\mathbf{A}=\left(\frac{1}{\Delta t} \mathbf{C}+\mathbf{K}\right)
\end{gathered}
$$

We can now construct a measure of the residual error with $\mathbf{T}_{m}$ and the measured temperature data to estimate the unknown parameters. However, $\mathbf{A}$ and $\mathbf{C}$ are so sparse that 
they lead to unnecessary matrix operations. To reduce the sparsity, Equation (3) can first be rearranged as follows:

$$
\begin{aligned}
{\left[\begin{array}{l}
\mathbf{T}_{v, m} \\
\mathbf{T}_{s, m}
\end{array}\right]=} & {\left[\begin{array}{ll}
\mathbf{A}_{v v} & \mathbf{A}_{v s} \\
\mathbf{A}_{s v} & \mathbf{A}_{s s}
\end{array}\right]^{-1} } \\
& \left(\frac{1}{\Delta t}\left[\begin{array}{ll}
\mathbf{C}_{v v} & \mathbf{C}_{v s} \\
\mathbf{C}_{s v} & \mathbf{C}_{s s}
\end{array}\right]\left[\begin{array}{l}
\mathbf{T}_{v, m-1} \\
\mathbf{T}_{s, m-1}
\end{array}\right]+\left[\begin{array}{l}
\mathbf{q}_{v, m} \\
\mathbf{q}_{s, m}
\end{array}\right]\right), \\
& \mathbf{A}_{v s}=\mathbf{A}_{s v}^{T}, \quad \mathbf{C}_{v s}=\mathbf{C}_{s v}^{T}
\end{aligned}
$$

where the subscript $v$ denotes virtual regions, which are quantities with respect to the unmeasured DOFs. The subscript $s$ denotes the regions corresponding to the measured DOFs by sensors. From Eq. (4a), $\mathbf{T}_{s, m}$ can then be defined as follows:

$$
\begin{gathered}
\mathbf{T}_{s, m}=\overline{\mathbf{A}}_{s s}^{-1}\left(\overline{\mathbf{C}}_{s s} \mathbf{T}_{s, m-1}+\overline{\mathbf{q}}_{s, m}\right) \\
\overline{\mathbf{q}}_{s, m}=\mathbf{q}_{s, m}-\mathbf{A}_{s v} \mathbf{A}_{v v}^{-1} \mathbf{q}_{v, m}+\overline{\mathbf{C}}_{s v} \mathbf{T}_{v, m-1}, \\
\overline{\mathbf{A}}_{s s}=\mathbf{A}_{s s}-\mathbf{A}_{s v} \mathbf{A}_{v v}^{-1} \mathbf{A}_{v s} \\
\overline{\mathbf{C}}_{s v}=\frac{1}{\Delta t}\left(\mathbf{C}_{s v}-\mathbf{A}_{s v} \mathbf{A}_{v v}^{-1} \mathbf{C}_{v v}\right) \\
\overline{\mathbf{C}}_{s s}=\frac{1}{\Delta t}\left(\mathbf{C}_{s s}-\mathbf{A}_{s v} \mathbf{A}_{v v}^{-1} \mathbf{C}_{v s}\right)
\end{gathered}
$$

where $\overline{\mathbf{A}}_{s s}$ is the Schur complement matrix [11], which is dense. From Eq. (4a), $\mathbf{T}_{v, m}$ also can then be defined as follows:

$$
\begin{gathered}
\mathbf{T}_{v, m}=\overline{\mathbf{A}}_{v v}^{-1}\left(\overline{\mathbf{C}}_{v v} \mathbf{T}_{v, m-1}+\overline{\mathbf{q}}_{v, m}\right) \\
\overline{\mathbf{q}}_{v, m}=\mathbf{q}_{v, m}-\mathbf{A}_{v s} \mathbf{A}_{s s}^{-1} \mathbf{q}_{s, m}+\overline{\mathbf{C}}_{v s} \mathbf{T}_{s, m-1}, \\
\overline{\mathbf{A}}_{v v}=\mathbf{A}_{v v}-\mathbf{A}_{v s} \mathbf{A}_{s s}^{-1} \mathbf{A}_{s v} \\
\overline{\mathbf{C}}_{v v}=\frac{1}{\Delta t}\left(\mathbf{C}_{v v}-\mathbf{A}_{v s} \mathbf{A}_{s s}^{-1} \mathbf{C}_{s v}\right) \\
\overline{\mathbf{C}}_{v s}=\frac{1}{\Delta t}\left(\mathbf{C}_{v s}-\mathbf{A}_{v s} \mathbf{A}_{s s}^{-1} \mathbf{C}_{s s}\right)
\end{gathered}
$$

Similarly, the unmeasured DOFs are divided into $i$ subdomains and can be decoupled by the Schur complement. Equation (6) can then be rewritten as

$$
\begin{aligned}
& \mathbf{T}_{v, m}=\tilde{\mathbf{A}}_{v v}^{-1}\left(\tilde{\mathbf{C}}_{v v} \mathbf{T}_{v, m-1}+\tilde{\mathbf{q}}_{v, m}\right) \\
& \mathbf{T}_{v, m}=\left[\begin{array}{llll}
{ }^{(1)} \mathbf{T}_{v, m}^{T} & { }^{(2)} \mathbf{T}_{v, m}^{T} & \ldots & { }^{(i)} \mathbf{T}_{v, m}^{T}
\end{array}\right]^{T},
\end{aligned}
$$

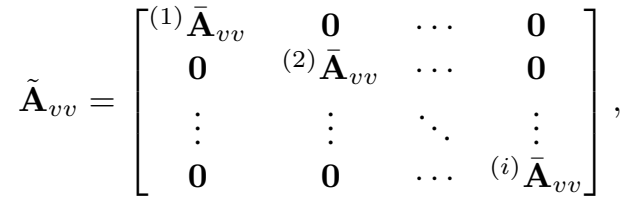

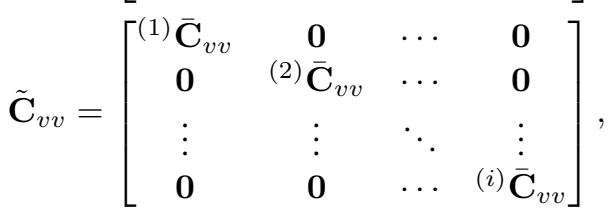

$$
\begin{aligned}
& \tilde{\mathbf{C}}_{v s}=\left[\begin{array}{llll}
{ }^{(1)} \overline{\mathbf{C}}_{v s}^{T} & { }^{(2)} \overline{\mathbf{C}}_{v s}^{T} & \ldots & { }^{(i)} \overline{\mathbf{C}}_{v s}^{T}
\end{array}\right]^{T},
\end{aligned}
$$

where the left superscript ${ }^{(i)}(\cdot)$ denotes the $i$-th subdomain, and ${ }^{(i)} \overline{\mathbf{A}}_{v v}$ is the $i$-th local Schur complement matrix except when $i=1$. Ideally, $\tilde{\mathbf{A}}_{v v}$ and $\tilde{\mathbf{C}}_{v v}$ can be diagonal matrices when the number of subdomains equals the number of unmeasured DOFs. The matrix operations in Eq. (4) can then be altered to the vector operations in Eq. (7). However, as the number of subdomains increases in Eq. (7), the computational cost of generating the system matrices is considerable. The costs of pre-processing and post-processing should be adjusted appropriately for this purpose.

The detailed solution algorithm for the VTS is provided in Section 1 , and we define $\mathbf{T}_{s, m}$ and $\mathbf{T}_{v, m}$ as $\overline{\mathbf{T}}_{m}$ and $\tilde{\mathbf{T}}_{m}$, respectively, for simplicity in the next Section.

\section{MODEL UPDATE}

This section introduces a method to calibrate the thermal conductivity, specific heat capacity, and thermal contact conductance. The thermal conductivity and specific heat capacity are tuned simultaneously, and the thermal contact conductance is calibrated in an assembled state. The thermal parameters can be updated by minimizing the squared residual errors, and the sum of the squared residual errors is defined as

$$
\begin{aligned}
S(\mathbf{P})=\sum_{m=1}^{M}\left[\mathbf{Y}_{m}-\overline{\mathbf{T}}_{m}(\mathbf{P})\right]^{T}\left[\mathbf{Y}_{m}-\overline{\mathbf{T}}_{m}(\mathbf{P})\right], \\
\mathbf{Y}_{m}=\left[\begin{array}{lll}
Y_{1, m} & \cdots & Y_{I, m}
\end{array}\right]^{T}, \\
\overline{\mathbf{T}}_{m}=\left[\begin{array}{lll}
T_{1, m} & \cdots & T_{I, m}
\end{array}\right]^{T}, \\
\mathbf{P}=\left[\begin{array}{lll}
P_{1} & \cdots & P_{N}
\end{array}\right]^{T},
\end{aligned}
$$

where $\mathbf{Y}_{m}$ and $\overline{\mathbf{T}}_{m}$ are the vectors containing the measured and calculated temperatures at a certain time step $m$, respectively, and the superscript $T$ indicates the transpose of the vector. $I, N$, and $M$ are the number of sensors, the number of unknown parameters, and the number of time steps, respectively.

To minimize $S(\mathbf{P})$, we implement an iterative process based on the Conjugate Gradient method [8], [16], and the iteration step consists of the following steps:

1) Initialization of unknown parameters.

2) Construct the finite element heat transfer model:

$$
\mathbf{C}^{(r)} \dot{\mathbf{T}}_{m}^{(r)}+\mathbf{K}^{(r)} \mathbf{T}_{m}^{(r)}=\mathbf{q}_{m}
$$

where superscript $(r)$ denotes the number of iterations of the CG method, and sets $r$ to 1 at first.

3) Determine the sensor information and compute the temperature using Eq. (5a).

4) Calculate the gradient direction by differentiating Eq. (8a) with respect to the unknown parameters:

$$
\left\{\nabla \mathbf{S}^{(r)}\right\}_{j}=-2 \sum_{m=1}^{M}\left\{\overline{\mathbf{X}}_{m}^{(r)}\right\}_{j}^{T}\left[\mathbf{Y}_{m}-\overline{\mathbf{T}}_{m}^{(r)}\right]=0
$$

where $\{\nabla \mathbf{S}(\mathbf{P})\}_{j}$ and $\left\{\overline{\mathbf{X}}_{m}\right\}_{j}, j=1, \cdots, N$, are the $j$-th gradient direction and the $j$-th sensitivity coefficient vector, respectively.

5) Compute the conjugation coefficient, which is given by the Fletcher-Reeves [31] expression in the form:

$$
\begin{array}{ll}
\gamma^{(r)}=\frac{\sum_{j=1}^{N}\left\{\nabla \mathbf{S}^{(r)}\right\}_{j}^{2}}{\sum_{j=1}^{N}\left\{\nabla \mathbf{S}^{(r-1)}\right\}_{j}^{2}}, & \text { for } \quad r=1,2, \cdots \\
\gamma^{(0)}=0 & \text { for } \quad r=0 .
\end{array}
$$


6) Calculate the direction of descent $\mathbf{d}^{(r)}$ :

$$
\mathbf{d}^{(r)}=\nabla \mathbf{S}^{(r)}+\gamma^{(r)} \mathbf{d}^{(r-1)} \quad \text { with } \quad \mathbf{d}^{(0)}=\mathbf{0} .
$$

7) Determine the search step size $\beta^{(r)}$ :

$$
\beta^{(r)}=\frac{\sum_{m=1}^{M}\left[\overline{\mathbf{X}}_{m}^{(r)} \mathbf{d}^{(r)}\right]^{T}\left[\overline{\mathbf{T}}_{m}^{(r)}-\mathbf{Y}_{m}\right]}{\sum_{m=1}^{M}\left[\overline{\mathbf{X}}_{m}^{(r)} \mathbf{d}^{(r)}\right]^{T}\left[\overline{\mathbf{X}}_{m}^{(r)} \mathbf{d}^{(r)}\right]}
$$

8) Unknown parameters are updated as:

$$
\mathbf{P}^{(r+1)}=\mathbf{P}^{(r)}-\beta^{(r)} \mathbf{d}^{(r)} .
$$

9) Increase $r$ by one and go to the second step.

\section{A. Thermal Conductivity and Specific Heat Capacity}

The thermal conductivity and the specific heat capacity are temperature-dependent parameters in practice. If the linearity of the material is strong within the operating temperature range of the machine, these two parameters can be assumed to be constant. In this case, the sensitivity coefficient matrix in Eq. (10) can be defined as follows:

$$
\begin{aligned}
\overline{\mathbf{X}}_{m}^{(r)} & =\mathbf{L}\left[\begin{array}{ll}
\frac{\partial \mathbf{T}_{m}^{(r)}}{\partial c_{v}} & \frac{\partial \mathbf{T}_{m}^{(r)}}{\partial k}
\end{array}\right], \\
& =\mathbf{L}\left[\begin{array}{ll}
\left.\mathbf{X}_{m}^{(r)}\right\}_{1} & \left\{\mathbf{X}_{m}^{(r)}\right\}_{2}
\end{array}\right],
\end{aligned}
$$

with

$$
\begin{gathered}
\left\{\mathbf{X}_{m}^{(r)}\right\}_{1}=\frac{1}{\Delta t} \mathbf{A}^{(r)^{-1}}\left[\mathbf{C}^{(r)}\left\{\mathbf{X}_{m-1}^{(r)}\right\}_{1}-\frac{\partial \mathbf{C}^{(r)}}{\partial c_{v}} \Delta \mathbf{T}_{m}^{(r)}\right] \\
\left\{\mathbf{X}_{m}^{(r)}\right\}_{2}=\mathbf{A}^{(r)^{-1}}\left[\frac{1}{\Delta t} \mathbf{C}\left\{\mathbf{X}_{m-1}^{(r)}\right\}_{2}-\frac{\partial \mathbf{K}^{(r)}}{\partial k} \mathbf{T}_{m}^{(r)}\right]_{(16 \mathrm{~b})} \\
\Delta \mathbf{T}_{m}^{(r)}=\left(\mathbf{T}_{m}^{(r)}-\mathbf{T}_{m-1}^{(r)}\right),
\end{gathered}
$$

where $\mathbf{L}$ is the Boolean matrix that maps the vector to the components corresponding to the sensor positions. The sizes of $\mathbf{L}, \mathbf{X}_{m}^{(r)}$, and $\overline{\mathbf{X}}_{m}^{(r)}$ are $\left(I \times N_{g}\right),\left(N_{g} \times N\right)$, and $(I \times N)$, respectively.

For computational efficiency, $\overline{\mathbf{X}}_{m}^{(r)}$ can be obtained using Eq. (5a) as follows:

$$
\begin{aligned}
& \overline{\mathbf{X}}_{m}^{(r)}=\left[\begin{array}{ll}
\frac{\partial \overline{\mathbf{T}}_{m}^{(r)}}{\partial c_{v}} & \frac{\partial \overline{\mathbf{T}}_{m}^{(r)}}{\partial k}
\end{array}\right], \\
& =\left[\begin{array}{ll}
\left\{\overline{\mathbf{X}}_{m}^{(r)}\right\}_{1} & \left\{\overline{\mathbf{X}}_{m}^{(r)}\right\}_{2}
\end{array}\right], \\
& \left\{\overline{\mathbf{X}}_{m}^{(r)}\right\}_{1}=\frac{1}{\Delta t} \overline{\mathbf{A}}_{s s}^{(r)^{-1}}\left[\overline{\mathbf{C}}_{s s}^{(r)}\left\{\overline{\mathbf{X}}_{m-1}^{(r)}\right\}_{1}-\frac{\partial \overline{\mathbf{C}}_{s s}^{(r)}}{\partial c_{v}} \Delta \overline{\mathbf{T}}_{m}^{(r)}\right], \\
& \left\{\overline{\mathbf{X}}_{m}^{(r)}\right\}_{2}=\overline{\mathbf{A}}_{s s}^{(r)^{-1}}\left[\frac{1}{\Delta t} \overline{\mathbf{C}}_{s s}\left\{\overline{\mathbf{X}}_{m-1}^{(r)}\right\}_{2}-\frac{\partial \overline{\mathbf{K}}^{(r)}}{\partial k} \overline{\mathbf{T}}_{m}^{(r)}\right], \\
& \overline{\mathbf{K}}_{s s}=\mathbf{K}_{s s}-\mathbf{K}_{s v} \mathbf{K}_{v v}^{-1} \mathbf{K}_{v s}, \\
& \Delta \overline{\mathbf{T}}_{m}^{(r)}=\left(\overline{\mathbf{T}}_{m}^{(r)}-\overline{\mathbf{T}}_{m-1}^{(r)}\right) \text {, }
\end{aligned}
$$

Note that $\overline{\mathbf{X}}_{m}^{(r)}$ in Eqs. (15) and (17a) are exactly the same.

\section{B. Thermal Contact Conductance}

The factors influencing the thermal contact conductance include contact pressure, interstitial material, surface deformation, surface roughness, waviness, and flatness [32], [33]. Since the quantification of these factors can only be measured in a statistical sense, it is very challenging to consider all. Therefore, we assume thermal contact conductance as a constant and estimate the constant based on the inverse heat conduction problem. To do this, the following assumptions are considered to address the inherent complexity of the thermal contact problems:

1) Contact surfaces are clean.

2) Only bulk material cases are considered, not thin-film material.

3) Contacting solids have isotropic thermal and physical properties.

4) Contact surfaces are not warped or broken in the operating temperature range, and thermal resistance changes due to increased temperature may be ignored.

5) The contact pressure and thermal interface material are uniformly applied in the defined contact surfaces.

The sensitivity coefficient matrix is now constructed to calibrate the value of the thermal contact conductance and is defined as

$$
\begin{aligned}
& \overline{\mathbf{X}}_{m}^{(r)}=\left[\begin{array}{llll}
\frac{\partial \overline{\mathbf{T}}_{m}^{(r)}}{\partial h^{(1)}} & \cdots & \frac{\partial \overline{\mathbf{T}}_{m}^{(r)}}{\partial h^{(j)}} & \cdots
\end{array}\right],
\end{aligned}
$$

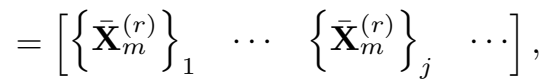

in which

$$
\left\{\overline{\mathbf{X}}_{m}^{(r)}\right\}_{j}=\overline{\mathbf{A}}_{s s}^{(r)^{-1}}\left[\frac{1}{\Delta t} \overline{\mathbf{C}}_{s s}^{(r)}\left\{\overline{\mathbf{X}}_{m-1}^{(r)}\right\}_{j}-\frac{\partial \overline{\mathbf{K}}_{s s}^{(r)}}{\partial h^{(j)}} \overline{\mathbf{T}}_{m}^{(r)}\right]_{(19)}
$$

where $h^{(j)}, j=1, \cdots, N_{c}$, is the thermal contact conductance specified on the $j$-th contact surface. $N_{c}$ denotes the number of contact surfaces.

\section{VIRTUAL THERMAL SENSOR}

\section{A. Virtual Thermal Sensing}

The updated numerical model described in the previous section is used to estimate the unknown heat source and temperature distribution. Such time-varying function estimation is obtained by minimizing the ordinary squares norm based on the sequential time-domain. The estimated solutions based on the sequential time-domain method are extremely sensitive to measurement errors, causing severe oscillation in estimated solutions. We use a Tikhonov regularization method to reduce this instability. Therefore, we incorporate the zerothorder regularization term into Eq. (8) and then obtain [7]

$$
\begin{aligned}
S_{m}=\left[\mathbf{Y}_{m}-\overline{\mathbf{T}}_{m}\right. & ]^{T}\left[\mathbf{Y}_{m}-\overline{\mathbf{T}}_{m}\right]+\alpha \Delta \mathbf{P}_{m}^{T} \Delta \mathbf{P}_{m}, \\
\Delta \mathbf{P}_{m} & =\mathbf{P}_{m}-\mathbf{P}_{m-1}, \\
\mathbf{P}_{m} & =\left[\begin{array}{lll}
\mathbf{g}_{m}^{T} & \mathbf{f}_{m}^{T}
\end{array}\right]^{T}, \\
\mathbf{g}_{m} & =\left[\begin{array}{llll}
g_{1, m} & \cdots & g_{k, m} & \cdots
\end{array}\right]^{T}, \\
\mathbf{f}_{m} & =\left[\begin{array}{llll}
f_{1, m} & \cdots & f_{l, m} & \cdots
\end{array}\right]^{T},
\end{aligned}
$$


where $g_{k, m}$ is the internal heat rate generated inside the $k$-th body at time $t_{m}$, and $f_{l, m}$ is the surface heat flux imposed on the $l$-th surface at time $t_{m} . S_{m}$ and $\overline{\mathbf{T}}_{m}$ are the functions of $\mathbf{P}_{m}$. The second term on the right-hand side of Eq. (20a) is a zeroth-order regularization term. $\alpha$ is a regularization parameter.

By solving the minimization problem in Eq. (20), the matrix normal equation and estimated solutions are derived as

$$
\begin{aligned}
\Delta \mathbf{P}_{m}=\left(\overline{\mathbf{X}}^{T} \overline{\mathbf{X}}+\alpha \mathbf{I}\right)^{-1} \overline{\mathbf{X}}^{T}\left[\mathbf{Y}_{m}-\hat{\mathbf{T}}_{s, m}\right], \\
\mathbf{P}_{m} \approx \mathbf{P}_{m-1}+\Delta \mathbf{P}_{m}, \\
\mathbf{T}_{m} \approx \hat{\mathbf{T}}_{m}+\mathbf{X} \Delta \mathbf{P}_{m}, \\
\hat{\mathbf{T}}_{m}=\left[\begin{array}{ll}
\hat{\mathbf{T}}_{v, m}^{T} & \hat{\mathbf{T}}_{s, m}^{T}
\end{array}\right]^{T}
\end{aligned}
$$

in which

$$
\begin{array}{r}
\hat{\mathbf{T}}_{v, m}=\tilde{\mathbf{A}}_{v v}^{-1}\left(\tilde{\mathbf{C}}_{v v} \mathbf{T}_{v, m-1}+\tilde{\mathbf{q}}_{v, m-1}\right), \\
\hat{\mathbf{T}}_{s, m}=\overline{\mathbf{A}}_{s s}^{-1}\left(\overline{\mathbf{C}}_{s s} \mathbf{T}_{s, m-1}+\overline{\mathbf{q}}_{s, m-1}\right), \\
\mathbf{X}=\tilde{\mathbf{A}}^{-1} \frac{\partial \mathbf{q}_{m}}{\partial \mathbf{P}_{m}}, \\
\overline{\mathbf{X}}=\mathbf{L X}, \quad \tilde{\mathbf{A}}=\left[\begin{array}{cc}
\tilde{\mathbf{A}}_{v v} & \mathbf{0} \\
\mathbf{0} & \overline{\mathbf{A}}_{s s}
\end{array}\right]
\end{array}
$$

where $\hat{\mathbf{T}}_{m}$ is the virtual temperature under a uniform load condition $\left(\mathbf{P}_{m}=\mathbf{P}_{m-1}\right)$, and $\mathbf{P}_{m}$ is the estimated heat source vector. $\mathbf{T}_{m}$ is the estimated temperature vector that contains information on the regions without sensors attached. $\left(\partial \mathbf{q}_{m} / \partial \mathbf{P}_{m}\right)$ is the unit heat flux matrix and does not change over time in linear problems (i.e., the thermal material properties are constants).

\section{B. Regularization Parameter for Stability}

In Eq. (21a), the regularization parameter $\alpha$ controls the degree of filtering of the amplified measurement noise. If the value of $\alpha$ is too large, a significant amount of information in the solution is lost. Therefore, it is important to select the optimal $\alpha$. In this work, we propose an efficient ridge estimator for the temperature estimation.

The efficient ridge estimator is based on the sum of the squares of the bias and variance errors. It is already well known that the Tikhonov regularization method [19], [20] is a bias-variance trade-off approach. The square of the total error can be calculated as

$$
\mathscr{T}^{2}(\alpha)=\mathscr{D}^{2}(\alpha)+\mathscr{V}^{2}(\alpha),
$$

where $\mathscr{T}, \mathscr{D}$ and $\mathscr{V}$ are the total, the bias, and the variance errors, respectively, and are functions of $\alpha$.

First, the square of the bias error is defined as

$$
\mathscr{D}^{2}=\frac{1}{M} \sum_{m=1}^{M}\left(\delta \mathbf{T}_{\text {bias }, m}\right)^{T}\left(\delta \mathbf{T}_{\text {bias }, m}\right),
$$

in which $\delta \mathbf{T}_{\text {bias, } m}$ is the bias error vector. To calculate the bias error vector, the gradient of Eq. (20a) is set to zero, which yields

$$
\alpha \Delta \mathbf{P}_{m}=\overline{\mathbf{X}}^{T}\left[\mathbf{Y}_{m}-\overline{\mathbf{T}}_{m}\right]
$$

where $\hat{\mathbf{T}}_{m}$ can be defined as

$$
\overline{\mathbf{T}}_{m}=\mathbf{Y}_{m}-\delta \mathbf{T}_{\text {bias }, m},
$$

Here, $\delta \mathbf{T}_{\text {bias, } m}$ is the bias error vector of the temperature corresponding to the sensor positions and is a function of $\alpha$. When $\alpha$ is zero, $\delta \mathbf{T}_{\text {bias }, m}$ becomes a zero vector, which indicates that $\hat{\mathbf{T}}_{m}$ is over-fitted to $\mathbf{Y}_{m}$. Substituting Eq. (26) into Eq. (25) gives

$$
\delta \mathbf{T}_{b i a s, m}=\alpha\left(\overline{\mathbf{X}} \overline{\mathbf{X}}^{T}\right)^{+} \overline{\mathbf{X}} \Delta \mathbf{P}_{m},
$$

where $\left(\mathbf{X}_{s} \mathbf{X}_{s}^{T}\right)^{+}$is the Moore-Penrose generalized inverse of $\mathbf{X}_{s} \mathbf{X}_{s}^{T}$. The inverse solution of the heat source tends to deviate initially and to become parallel to the exact solution. Using this characteristic, we assume that $\Delta \mathbf{P}_{m}$ equals the exact heat source change when ignoring the deviated error. We then consider only the maximum heat source change to calculate the maximum bias error. Note that this assumption can be used when the sensors are attached to certain positions with high sensitivity coefficients. Therefore, the equations (24) and (27) can be redefined as

$$
\begin{gathered}
\mathscr{D}^{2}=\left(\delta \mathbf{T}_{\text {bias }}\right)^{T}\left(\delta \mathbf{T}_{\text {bias }}\right), \\
\delta \mathbf{T}_{\text {bias }}=\alpha\left(\overline{\mathbf{X}} \overline{\mathbf{X}}^{T}\right)^{+} \overline{\mathbf{X}} \Delta \mathbf{P}_{\text {max }}, \\
\Delta \mathbf{P}_{\text {max }}:=\left\{\Delta \mathbf{P}_{m}\right\}_{\text {max }}, \quad \text { for } \quad 1 \leq m \leq M .
\end{gathered}
$$

Second, the square of the variance error is defined as

$$
\mathscr{V}^{2}=\frac{1}{M} \sum_{m=1}^{M}\left(\delta \mathbf{T}_{v a r, m}\right)^{T}\left(\delta \mathbf{T}_{v a r, m}\right),
$$

where $\delta \mathbf{T}_{v a r, m}$ is a variance error vector of the temperature. To calculate the propagation and accumulation of the measurement error over time, Equation (21a) can be rewritten using $\mathbf{L} \mathbf{T}_{m}$ instead of $\overline{\mathbf{T}}_{m}$, as follows:

$$
\begin{gathered}
\Delta \mathbf{P}_{m}=\mathbf{G}\left[\mathbf{Y}_{m}-\mathbf{L}\left\{\mathbf{B}^{m} \mathbf{T}_{0}+\left(\sum_{p=0}^{m-1} \mathbf{B}^{p} A^{-1} \mathbf{q}_{0}\right)\right.\right. \\
\left.\left.+\left(\sum_{i=1}^{m-1} \sum_{k=0}^{i} \mathbf{B}^{k} \mathbf{X} \Delta \mathbf{P}_{m-i}\right)\right\}\right], \\
\mathbf{G}=\left(\overline{\mathbf{X}}^{T} \overline{\mathbf{X}}+\alpha \mathbf{I}\right)^{-1} \overline{\mathbf{X}}^{T}, \\
\mathbf{B}=\frac{1}{\Delta t} \mathbf{A}^{-1} \mathbf{C},
\end{gathered}
$$

where $\mathbf{T}_{0}$ and $\mathbf{q}_{0}$ are the initial temperature and thermal loading vectors, respectively. The initial errors of $\mathbf{T}_{0}$ and $\mathbf{q}_{0}$ are negligible if the thermal boundary condition is clearly defined with a well-established experimental environment. Equation (30a) could then be defined using only the measurement error:

$$
\begin{gathered}
\delta \Delta \mathbf{P}_{v a r, m}=\mathbf{G}\left[\delta \mathbf{Y}_{m}-\mathbf{L} \sum_{i=1}^{m-1}\left(\mathbf{I}-\mathbf{B}^{i+1}\right) \mathbf{D} \delta \mathbf{Y}_{m-i}\right] \\
\mathbf{D}=(\mathbf{I}-\mathbf{B})^{-1} \mathbf{X G}
\end{gathered}
$$

where $\delta \Delta \mathbf{P}_{\text {var, }}$ is the variance error of the heat source change. $\delta \mathbf{Y}^{m+1}$ is the measurement error vector and is normally distributed with zero mean and standard deviation, 
denoted by $\delta \mathbf{Y}^{m+1} \sim \mathcal{N}\left(\mathbf{0}, \boldsymbol{\sigma}^{2}\right)$, where $\boldsymbol{\sigma}$ is an $I$-dimensional standard deviation (i.e., the measurement noise level of the sensors) vector.

The summation term in Eq. (31) represents the propagation and accumulation of errors, and it entails high computational resources. To solve this problem, we assume $\delta \mathbf{Y}_{m}$ as a regular oscillation:

$$
\delta \mathbf{Y}_{m}=(-1)^{m} \boldsymbol{\sigma} .
$$

By substituting Eq. (32) into Eq. (31), we can obtain the time-independent variance error of the heat source change:

$$
\delta \Delta \mathbf{P}_{v a r}=\mathbf{G}\left[\mathbf{I}+\mathbf{L}\left(\mathbf{I}+\mathbf{B}^{M_{a}}\right)(\mathbf{I}+\mathbf{B})^{-1} \mathbf{B}^{2} \mathbf{D}\right] \boldsymbol{\sigma},
$$

where $\delta \Delta \mathbf{P}_{\text {var }}$ is the vector of the time-independent variance error of the heat source. $M_{a}$ is a time step at the moment when the norm of $\mathbf{A}^{\left(M_{a}\right)}$ is less than one. Equation (33) is derived when $M_{a}$ is odd, and in a similar manner, the equation can be derived when $M_{a}$ is even.

Equation (21c) indicates that the variance error of the temperature is determined by the variance error of the heat source change. To compensate for the assumption in Eq. (32), the variance error of the temperature can be defined as follows:

$$
\begin{gathered}
\delta \mathbf{T}_{\text {var }}=\xi(\alpha) \delta \Delta \mathbf{P}_{\text {var }}, \\
\xi(\alpha)=\sqrt{\frac{\sum_{m=1}^{M_{s}}\left|\delta \mathbf{T}_{s, m}\right|^{2}}{\sum_{m=1}^{M_{s}}\left|\delta \Delta \mathbf{P}_{m}\right|^{2}}},
\end{gathered}
$$

where $\delta \mathbf{T}_{s, m}$ and $\delta \Delta \mathbf{P}_{m}$ are the temperature and the heat source change vectors with zero mean, respectively, and are calculated by $\delta \mathbf{Y}_{m}$, which is the measurement noise data with zero mean. Using Eq. (34), Equation (29) can be redefined as

$$
\mathscr{V}^{2}=\left(\delta \mathbf{T}_{v a r}\right)^{T}\left(\delta \mathbf{T}_{v a r}\right) .
$$

Finally, the total error of the temperature is calculated as the sum of $\mathscr{D}^{2}$ and $\mathscr{V}^{2}$ defined in Eqs. (28a) and (35), respectively. Note that Eqs. (28a) and (35) do not depend on the temperature history, and thus, this approach can be utilized for the initial estimation without collecting measurement data in advance. It is also important that the bias error is a function of the heat source change and $\alpha$, and the variance error is a function of the measurement noise and $\alpha$.

\section{Algorithm}

This Section introduces the algorithm for constructing the processing unit of the VTS by organizing the contents of the preceding Section. The algorithm is presented below.

The model update process is not included in this algorithm. The sequence of virtual thermal sensing first estimates the heat source and predicts the temperature distribution using the estimated heat source. $\mathbf{q}_{\kappa}$ is the thermal loading vector by the natural convection, and $N_{s d}$ is the number of subdomains. The coefficient matrix is calculated once during initialization to minimize the computational cost of the virtual sensing that occurs at every sampling time step. Note that the for loop on line 15 of the algorithm can be performed in parallel.

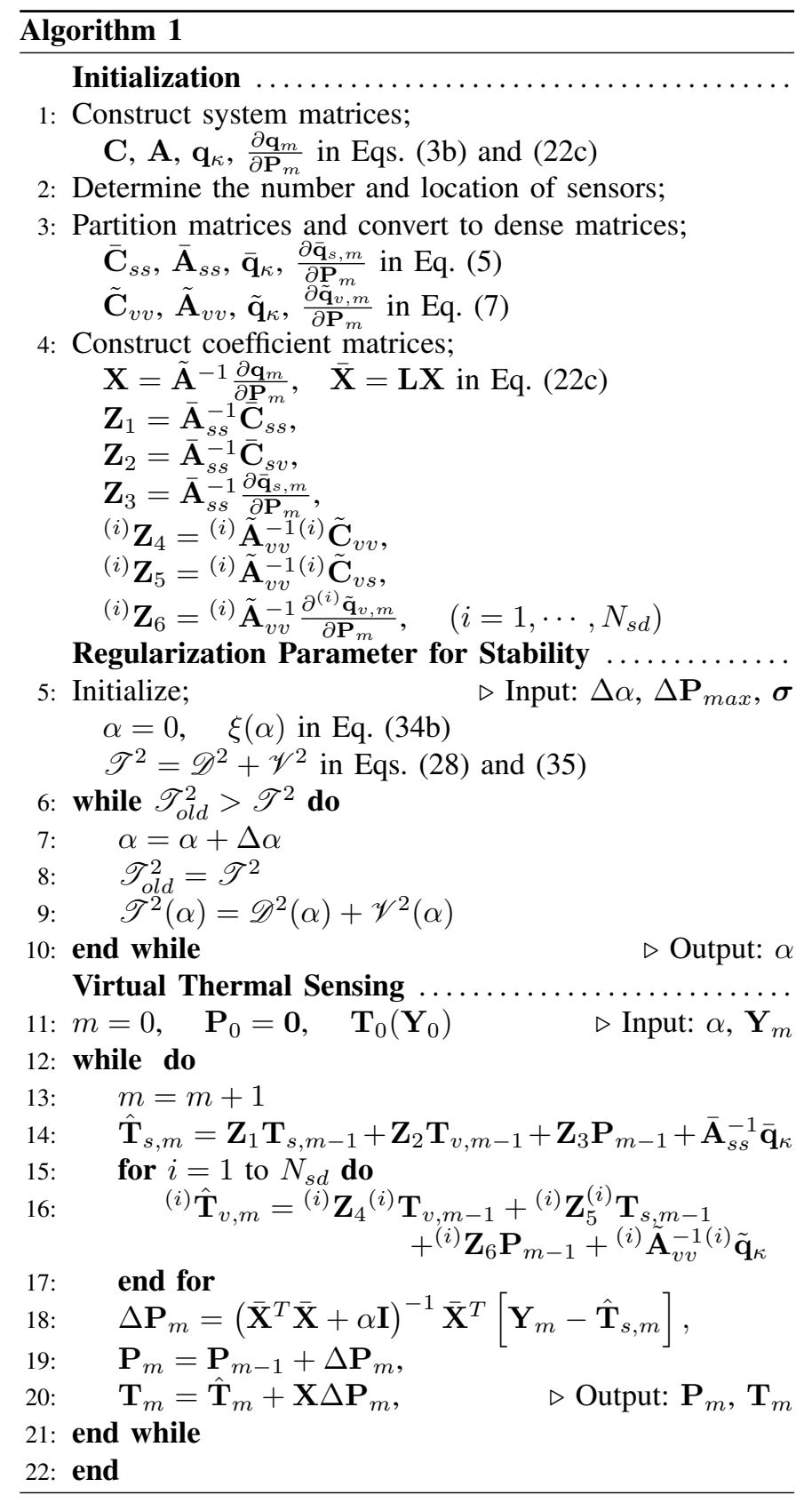

\section{EXPERIMENTAL RESULTS}

In this Section, the proposed model-based virtual thermal sensor is experimentally validated using a sealed cylindrical structure in which a commercial servo drive operates. The cylindrical structure comprises three parts that are assembled by the thermal contact: the middle and bottom parts are made of aluminum $6061 \mathrm{~T}-6$, and the top part is made of 303 stainless steel. A commercial servo drive is located inside this structure with the heat-sink as illustrated in Figure 1 and is used to control the EC motor, brushless, $400 \mathrm{~W}$. We utilized a Gold Solo Twitter (Elmo Motion Control, Petah Tikva, Israel) that delivers up to $5 \mathrm{~kW}$ power in an average $1.87 \mathrm{in}^{3}$ compact package, and a flat heat-sink provided by Elmo Inc. was used to dissipate the heat. The Gold Solo Twitter (GTWI) has six MOSFETs located on the metal PCB, shown in Figure 1b. It is necessary to monitor the temperature of the MOSFET 
because most of the heat is generated there. However, it is not easy to attach the temperature sensor directly. Even if a sensor can be inserted inside, the GTWI is designed to be compact, making it difficult to attach the bead of the sensor directly to the MOSFET. Therefore, we use a virtual thermal sensor to estimate the temperature and heat source of the MOSFET without the aid of a sensor installed inside.

\section{A. Experimental Set-up and Numerical Modeling}

This study strictly controls the experimental environment to eliminate unpredictable error factors. First, the heat dissipation by convection and jig should be well established. Convection is the source of non-white noise, which is difficult to filter. Therefore, it is desirable to remove the effects of convection using a vacuum chamber; thus, we utilized the chamber and vacuum pump in Figure 2. In all the experiments, the degree of vacuum and the chamber's internal (= ambient) temperature are set at $100 \mathrm{mbar}$ and about $50{ }^{\circ} \mathrm{C}$, respectively. In these chamber conditions, the convective heat transfer coefficient, $\tau$, is about $6.16 \mathrm{~W} / \mathrm{m}^{2} K$ [34], [35]. We minimize the heat dissipation by the jig using a thread with low thermal conductivity, as illustrated in Figure 3. T-type thermocouples (CopperConstantan) were used, and the sampling rate is $2 \mathrm{~Hz}$ for all experiments. This type of thermocouple (TC) has a continuous temperature range from -250 up to $350{ }^{\circ} \mathrm{C}$ and maximum error of $\pm 1.0^{\circ} \mathrm{C}$ or $\pm 0.75 \%$. For the numerical modeling of the VTS, we consider the three-dimensional heat transfer problem and implement finite element modeling with eightnode hexahedral elements. The information for the FE models is listed in TABLE II, and herein, the DOF per node is one. The experimental setup conditions for the model update process and the virtual sensing are listed in TABLE III.

\section{B. Offline Model Update}

The model update process was performed to minimize modeling errors. The model parameters were tuned in the range of $50^{\circ}$ to $65^{\circ} \mathrm{C}$ considering the operating temperature range of the housing. After calibrating the thermal conductivity and the specific heat capacity before assembly, the thermal contact conductance was tuned by assembling. The experimental setup for tunning the two parameters is illustrated in Figures 3 and 4, and the temperature results before and after tuning are shown in Figures $6 \mathrm{a}$ and $7 \mathrm{a}$. The mean squared error (MSE) of the sensors is adopted to evaluate the performance of the model update and is defined as

$$
(M S E)_{m}=\sum_{i=1}^{I}\left[Y_{i, m}-T_{i, m}\right]^{T}\left[Y_{i, m}-T_{i, m}\right],
$$

where $i$ and $m$ denote the sensor number and the time step. Figures $6 \mathrm{~b}$ and $7 \mathrm{~b}$ are the results of the MSE. The initial and updated values of the two parameters for each material are listed in TABLE IV, and the typical values of each material are also indicated. Note that material properties are depend on the manufacturing quality. Specifically, there may be a significant difference between the typical and updated values. The results in the middle part indicated similar results to those of the bottom made of the same material. The experimental setting for updating the thermal contact conductance is illustrated in Figures 5. The temperature result, the MSE, and the updated values of the thermal contact conductance are shown in Figure 8 and TABEL V, respectively. Figures 6a, 7a, and 8a show only the temperature results of the two thermocouples for a clear comparison, and the results of the remaining thermocouples can be evaluated by the MSE.

\section{Online Virtual Thermal Sensing}

The updated model is utilized to construct the processing unit of the VTS. Algorithm 1 is programmed using MATLAB software on a processing unit equipped with Intel $17-8700 \mathrm{~K}$ CPU@3.70GHz and 64.0GB RAM. As a result of implementing the Algorithm with this processor, the computational cost is shown in Figure 12. The processing time per time step is the time required for $m$ to increase by one on line 13 of Algorithm 1. In the legend, $i$ denotes the number of subdomains decoupled by the Schur complement, and thus, $(i=6)$ implies that the system matrices are decoupled into six subdomains: top, middle, bottom, heat-sink, PCB, and MOSFET. $(i=1)$ has $323,100,625(=17,975 \times 17,975)$ elements in the matrix, but $(i=6)$ has $58,788,463$ elements in the matrix. EtherCAT protocol is utilized for communication between devices. The chamber conditions are similar to the previous experiments, and the GTWI drives the motor at 2500 $\mathrm{rpm}$ in a torque-controlled environment at $0.843 \mathrm{Nm}$. At this time, the continuous output current of the GTWI is $8 A$ to $8.5 A$, and the bus voltage is $24 V D C$. Elmo Inc. provides the information that the control of the GTWI consumes 2.5 $W$ to $4 W$, depending on the encoder's loading and on the type of communication and the GTWI is designed such that the maximum heat-sink temperature should be no higher than $80^{\circ} \mathrm{C}$ to $85^{\circ} \mathrm{C}$.

One thermocouple (TC 16) is used for virtual sensing, and the location of the sensor is illustrated in Figure 9a. Temperature data measured at this thermocouple corresponds to $\mathbf{Y}_{m}$ in Eq. (21a). The regularization parameter $\alpha$ used for stability is $2.16 \times 10^{-17}$, obtained by the proposed ridge estimator in Section IV-B. The optimal $\alpha$ value selected by the proposed estimator is compared with the Morozov discrepancy principle to prove that it is reasonable. The results are shown in Figure 10 and indicate that the optimal $\alpha$ values estimated by the two methods are similar. The time required for each method to estimate the optimal $\alpha$ is 16.13 hours and 1.29 hours, respectively. First, we estimate the heat source of the MOSFET using the VTS with one thermocouple (TC 16) attached to the floor. Figure $11 \mathrm{~b}$ shows the estimated heat source of MOSFET, which is the result obtained by substituting the measured data $\mathbf{Y}_{m}$ for Eq. (21a), and Figure 11a illustrates the calculated temperature $\mathbf{T}_{s, m}$. In addition to $\mathbf{T}_{s, m}$, the temperature distribution of the unmeasured position is predicted, and the results are shown in Figure 13. The image in Figure $13 \mathrm{~b}$ is captured by an infrared (IR) camera, Testo 871. An IR camera cannot obtain an internal temperature, but the VTS address this challenge and monitor the temperature of the MOSFET. 
Finally, four thermocouples (TC $12 \sim 15$ ) are used to prove that the estimated temperature distribution by the VTS is reliable. Three thermocouples (TC $13 \sim 15$ ) are attached to the housing, one to the heat sink (TC 12), and the locations of attachment are illustrated in Figures 5a and 9a. The results of physical sensing by thermocouples (TC 12 15) and virtual sensing by the proposed VTS are shown in Figure 14, where it can be seen that virtual sensing follows physical sensing well. It is also confirmed using the virtual thermal sensor that the MOSFET operates within the upper temperature limit of design specifications $\left(80^{\circ} \sim 85^{\circ} \mathrm{C}\right)$.

\section{CONCLUSION}

This study developed a virtual thermal sensor for the realtime monitoring of electronic packages with sensor attachment limitations. Minimizing modeling errors and measurement errors were the most important in developing reliable VTS, and real-time virtual sensing requires programming algorithms that can be implemented using affordable processors. A finite element model and its updating technique were used to minimize the uncertainties of the thermal parameters that arise during the manufacturing process. The computational burden caused by the consistent matrices of the finite element model was reduced using the Schur complement technique. This implementation was performed with six subdomains, which saved the processing time per time interval up to four times. In addition, the Tikhonov regularization method was utilized to reduce the sensitivity to measurement errors, and we proposed an estimator for efficiently selecting the regularization parameter. The proposed estimator was verified by comparing it with the widely used Morozov discrepancy principle. The time required for each method to estimate the optimal $\alpha$ was 16.13 hours and 1.29 hours, respectively, demonstrating efficiency.

Finally, the proposed VTS was implemented and experimentally evaluated with an enclosed structure in which the commercial servo drive, Gold Solo Twitter, operates. The VTS monitored that MOSFETs, the main heat source of Gold Solo Twitter, operates near $85^{\circ} \mathrm{C}$, generating about $2.5 \mathrm{~W}$ of heat. The advantage of the VTS is that it can estimate the amount of heat generated without electrical knowledge. In addition, taking advantage of the finite element method, the proposed VTS can also be installed in systems with more complicated heat flows. Therefore, the proposed VTS can be sufficiently applied to other applications, such as robot actuators, electric vehicle batteries, electric motors, semiconductor manufacturing thermal processors, and transformers.

\section{REFERENCES}

[1] Y. Xiao, "Model-based virtual thermal sensors for lithium-ion battery in ev applications," IEEE Transactions on Industrial Electronics, vol. 62, no. 5, pp. 3112-3122, 2014.

[2] R. Modirnia and B. Boulet, "Model-based virtual sensors and coretemperature observers in thermoforming applications," IEEE Transactions on Industry Applications, vol. 49, no. 2, pp. 721-730, 2013.

[3] R. Saheba, M. Rotea, O. Wasynczuk, S. Pekarek, and B. Jordan, "Virtual thermal sensing for electric machines," IEEE Control Systems Magazine, vol. 30, no. 1, pp. 42-56, 2010.

[4] P. N. Phuc, D. V. Bozalakov, H. Vansompel, K. Stockman, and G. Crevecoeur, "Rotor temperature virtual sensing for induction machines using a lumped-parameter thermal network and dual kalman filtering," IEEE Transactions on Energy Conversion, 2021.
[5] S. Oh, D. Park, H. Baek, S. Kim, J. Lee, and J. Kim, "Virtual sensing system of structural vibration using digital twin," Trans. Korean Soc. Noise \& Vib. Eng, vol. 30, no. 2, pp. 149-160, 2020.

[6] S. Oh, K. Lee, G. Ahn, K. Kim, and J. Kim, "Development of vibroacoustic virtual sensing system for pipeline structure," Transactions of the KSME A, pp. 254-255, 2021.

[7] J. V. Beck, B. Blackwell, and C. R. S. Clair Jr, Inverse heat conduction: Ill-posed problems. James Beck, 1985.

[8] M. N. Özisik and H. R. Orlande, Inverse heat transfer: fundamentals and applications. CRC press, 2021.

[9] K.-J. Bathe, Finite element procedures. Klaus-Jurgen Bathe, 2006.

[10] S. Babadi and K. Ebrahimi, "Real time monitoring of long slender shaft by distributed-lumped modeling techniques," International Journal of Electrical and Computer Engineering, vol. 6, no. 8, pp. 820-825, 2012.

[11] F. Zhang, The Schur complement and its applications, vol. 4. Springer Science \& Business Media, 2006.

[12] H. Ahmadian, J. Mottershead, and M. Friswell, "Regularisation methods for finite element model updating," Mechanical Systems and Signal Processing, vol. 12, no. 1, pp. 47-64, 1998.

[13] J. E. Mottershead, M. Link, and M. I. Friswell, "The sensitivity method in finite element model updating: A tutorial," Mechanical systems and signal processing, vol. 25, no. 7, pp. 2275-2296, 2011.

[14] F. Mohebbi, M. Sellier, and T. Rabczuk, "Estimation of linearly temperature-dependent thermal conductivity using an inverse analysis," International Journal of Thermal Sciences, vol. 117, pp. 68-76, 2017.

[15] C.-H. Huang and Y. Jan-Yuan, "An inverse problem in simultaneously measuring temperature-dependent thermal conductivity and heat capacity," International Journal of Heat and Mass Transfer, vol. 38, no. 18, pp. 3433-3441, 1995.

[16] Y. Jarny, M. Ozisik, and J. Bardon, "A general optimization method using adjoint equation for solving multidimensional inverse heat conduction," International journal of heat and mass transfer, vol. 34, no. 11, pp. 2911-2919, 1991.

[17] M. Asif, A. Tariq, and K. M. Singh, "Estimation of thermal contact conductance using transient approach with inverse heat conduction problem," Heat and Mass Transfer, vol. 55, no. 11, pp. 3243-3264, 2019.

[18] H. Orlande and M. Ozisik, "Inverse problem of estimating interface conductance between periodically contacting surfaces," Journal of thermophysics and heat transfer, vol. 7, no. 2, pp. 319-325, 1993.

[19] A. N. Tikhonov, "On the solution of ill-posed problems and the method of regularization," in Doklady Akademii Nauk, vol. 151, no. 3, pp. 501504. Russian Academy of Sciences, 1963.

[20] A. N. Tikhonov, A. Goncharsky, V. Stepanov, and A. G. Yagola, Numerical methods for the solution of ill-posed problems, vol. 328. Springer Science \& Business Media, 2013.

[21] O. M. Alifanov, Inverse heat transfer problems. Springer Science \& Business Media, 2012.

[22] J. V. Beck and D. A. Murio, "Combined function specificationregularization procedure for solution of inverse heat conduction problem," AIAA journal, vol. 24, no. 1, pp. 180-185, 1986.

[23] C. Groetsch, "Comments on morozov's discrepancy principle," in Improperly posed problems and their numerical treatment, pp. 97-104. Springer, 1983.

[24] H. Engl, "Discrepancy principles for tikhonov regularization of ill-posed problems leading to optimal convergence rates," Journal of optimization theory and applications, vol. 52, no. 2, pp. 209-215, 1987.

[25] G. H. Golub, M. Heath, and G. Wahba, "Generalized cross-validation as a method for choosing a good ridge parameter," Technometrics, vol. 21, no. 2, pp. 215-223, 1979.

[26] M. A. Lukas, "Robust generalized cross-validation for choosing the regularization parameter," Inverse Problems, vol. 22, no. 5, p. 1883 , 2006.

[27] D. M. Trujillo and H. R. Busby, "Optimal regularization of the inverseheat conduction problem using the 1-curve," International Journal of Numerical Methods for Heat \& Fluid Flow, 1994.

[28] P. C. Hansen and D. P. O'Leary, "The use of the 1-curve in the regularization of discrete ill-posed problems," SIAM journal on scientific computing, vol. 14, no. 6, pp. 1487-1503, 1993.

[29] K. A. Woodbury and J. V. Beck, "Estimation metrics and optimal regularization in a tikhonov digital filter for the inverse heat conduction problem," International Journal of Heat and Mass Transfer, vol. 62, pp. 31-39, 2013.

[30] C. Ahn, C. Park, D. Park, and J. Kim, "Optimal hybrid parameter selection for stable sequential solution of inverse heat conduction problem," 2021. 
TABLE I: NUMERICAL MODEL AND THERMAL PARAMETERS

\begin{tabular}{lcclcc}
\hline \multicolumn{1}{c}{ Numerical Model } & \multicolumn{4}{c}{ Thermal Parameters } \\
\hline $\begin{array}{l}\text { Capacity } \\
\text { matrix }\end{array}$ & $\mathbf{C}\left(\rho, c_{v}\right)$ & $\rho$ & density & $\mathrm{kg} / \mathrm{m}^{3}$ \\
& & $c_{v}$ & specific heat capacity & $\mathrm{J} / \mathrm{kg} \mathrm{K}$ \\
Heat transfer & $\mathbf{K}(k, h, \kappa)$ & $h$ & contact conductance & $\mathrm{W} / \mathrm{m}^{2} \mathrm{~K}$ \\
matrix & & $\kappa$ & convective coefficient & $\mathrm{W} / \mathrm{m}^{2} \mathrm{~K}$ \\
Thermal & & $\mathbf{g}$ & heat generation rate & $W / \mathrm{m}^{3}$ \\
loading vector & $\mathbf{q}(\mathbf{g}, \mathbf{f}, \kappa)$ & $\mathbf{f}$ & surface heat fluxes & $\mathrm{W} / \mathrm{m}^{2}$ \\
Temperature & $\mathbf{T}$ & $T$ & temperature & $\mathrm{K}$ \\
\hline
\end{tabular}

TABLE II: INFORMATION OF FINITE ELEMENT MODELS

\begin{tabular}{ccccc}
\hline Case & $\begin{array}{c}\text { \# of } \\
\text { nodes }\end{array}$ & $\begin{array}{c}\text { \# of } \\
\text { elements }\end{array}$ & $\begin{array}{c}\text { \# of } \\
\text { components }\end{array}$ & $\begin{array}{c}\text { Element } \\
\text { type }\end{array}$ \\
\hline Top & 3,435 & 2,184 & 1 & \\
Middle & 2,025 & 1,200 & 1 & \\
Bottom & 3,753 & 2,426 & 1 & Hexahedral \\
Heat-sink & 4,152 & 2,931 & 1 & element \\
PCB & 2,990 & 1,940 & 1 & \\
MOSFET & 270 & 144 & 6 & \\
\hline Total & 17,975 & 11,545 & 11 & \\
\hline
\end{tabular}

[31] R. Fletcher and C. M. Reeves, "Function minimization by conjugate gradients," The computer journal, vol. 7, no. 2, pp. 149-154, 1964.

[32] M. M. Nezhad, M. H. Shojaeefard, and S. Shahraki, "Studying the transient thermal contact conductance between the exhaust valve and its seat using the inverse method," International Journal of Thermophysics, vol. 37, no. 2, p. 13, 2016.

[33] D. Zhao, X. Qian, X. Gu, S. A. Jajja, and R. Yang, "Measurement techniques for thermal conductivity and interfacial thermal conductance of bulk and thin film materials," Journal of Electronic Packaging, vol. 138, no. 4, p. 040802, 2016.

[34] M. Saidi and R. H. Abardeh, "Air pressure dependence of naturalconvection heat transfer," in Proceedings of the World Congress on Engineering, vol. 2, no. 1, pp. 1-5. WCE 2010 London, UK, 2010.

[35] R. Hosseini and M. Saidi, "Experimental study of air pressure effects on natural convection from a horizontal cylinder," Heat transfer engineering, vol. 33, no. 10 , pp. 878-884, 2012.

TABLE III: EXPERIMENTAL SETUP CONDITIONS IN EACH PROCESS

\begin{tabular}{|c|c|c|c|c|}
\hline Case & $\begin{array}{c}\text { Unknown } \\
\text { Param }\end{array}$ & $\begin{array}{c}\text { \# of } \\
\text { sensors }\end{array}$ & Heat source & Note \\
\hline $\begin{array}{l}\text { Model update } \\
\text { (Top) }\end{array}$ & $k, c_{v}$ & $\begin{array}{c}3 \\
(\mathrm{TC} 1 \sim 3)\end{array}$ & $\begin{array}{c}\text { Halogen } \\
\text { lamp }\end{array}$ & $\begin{array}{l}* \text { Degree of } \\
\text { vacuum }\end{array}$ \\
\hline $\begin{array}{l}\text { Model update } \\
\text { (Bottom) }\end{array}$ & $k, c_{v}$ & $\begin{array}{c}3 \\
\text { (TC4 6) }\end{array}$ & $\begin{array}{c}\text { Halogen } \\
\text { lamp }\end{array}$ & $\begin{array}{l}: 100 \text { mbar } \\
* \text { Sampling }\end{array}$ \\
\hline $\begin{array}{l}\text { Model update } \\
\text { (Assembly) }\end{array}$ & $h$ & $\begin{array}{c}6 \\
(\mathrm{TC} 7 \sim 12)\end{array}$ & $\begin{array}{c}\text { Silicone } \\
\text { heater }\end{array}$ & $\begin{array}{l}\text { rate: } 2 \mathrm{~Hz} \\
\text { *Ambient }\end{array}$ \\
\hline $\begin{array}{c}\text { Virtual } \\
\text { sensing }\end{array}$ & $\mathrm{g}$ & $\begin{array}{c}1 \\
(\mathrm{TC} 16)\end{array}$ & $\begin{array}{l}\text { MOSFET } \\
\text { (unknown) }\end{array}$ & $\begin{array}{l}\text { temperature } \\
\text { about } 50^{\circ} \mathrm{C}\end{array}$ \\
\hline
\end{tabular}

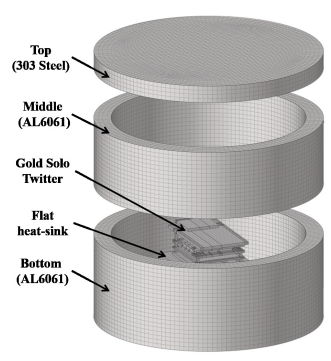

(a)

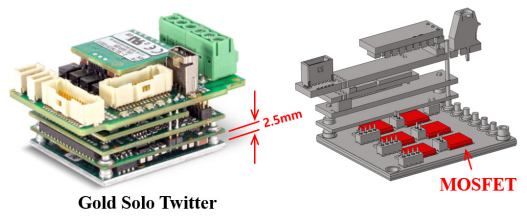

(b)
Fig. 1: (a) Finite element model of each component, and (b) Gold Solo Twitter has six MOSFETs located on a metal PCB.

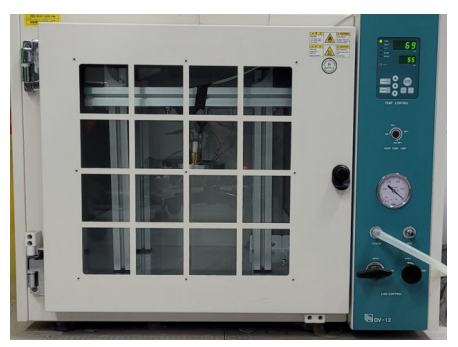

(a)

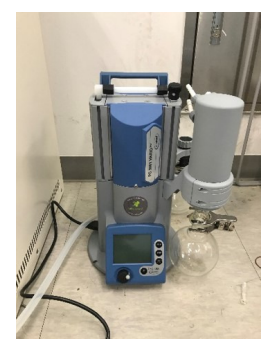

(b)
Fig. 2: (a) Chamber and (b) vacuum pump. In all experiments, the degree of vacuum and internal temperature of the chamber were set at $100 \mathrm{mbar}$ and $50^{\circ} \mathrm{C}$, respectively.

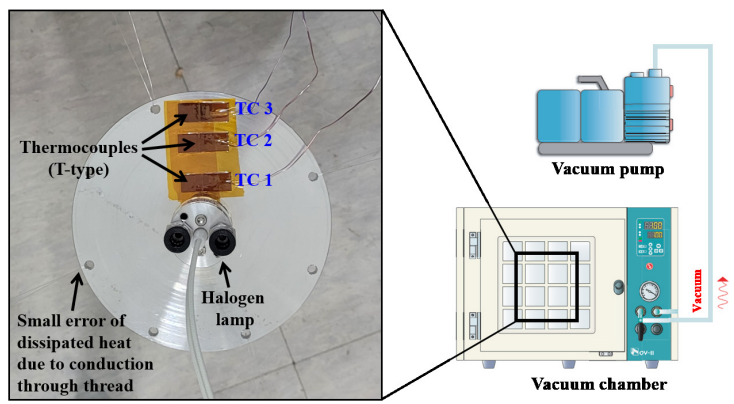

Fig. 3: Experimental boundary conditions and sensor locations for model update process of Top part.

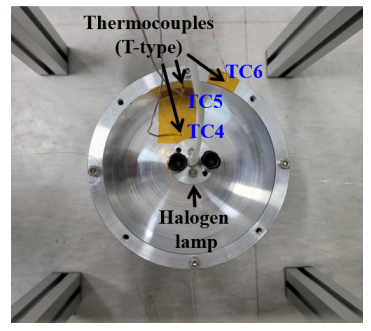

(a)

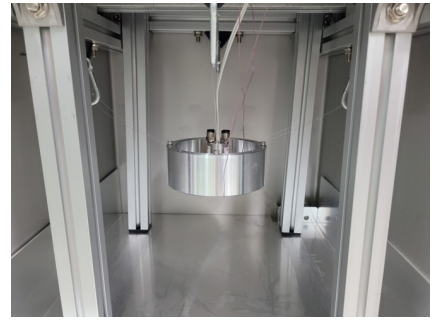

(b)
Fig. 4: Experimental boundary conditions and sensor locations for model update process of Bottom part. 


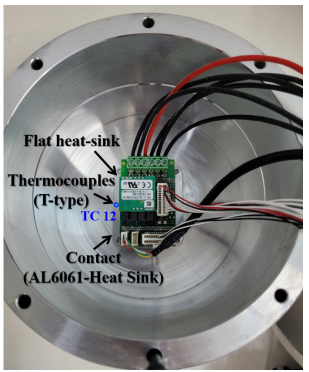

(a)

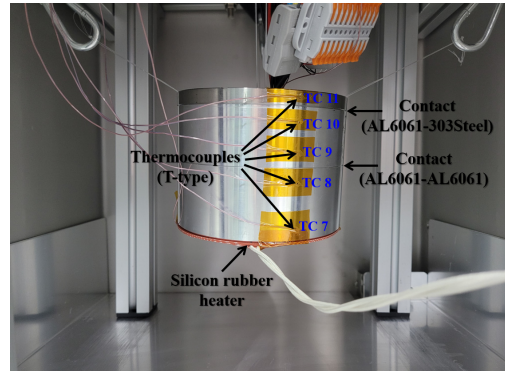

(b)
Fig. 5: Experimental boundary conditions and sensor locations to update the thermal contact conductance of the three contact surfaces.

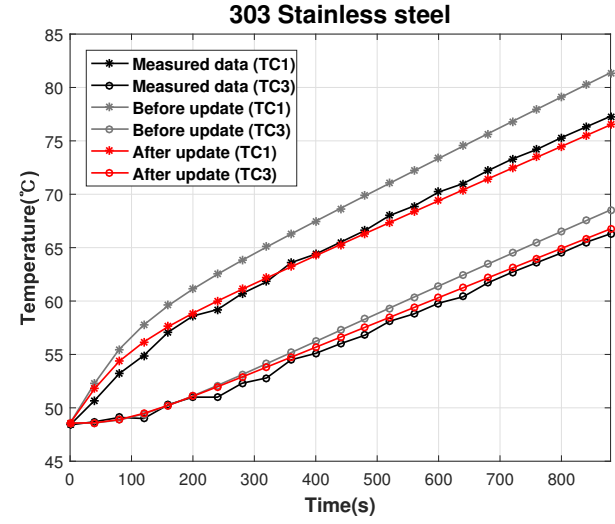

(a)

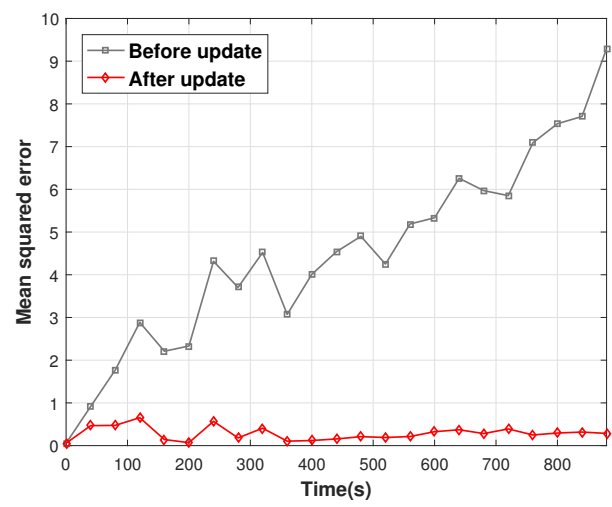

(b)

Fig. 6: Comparison of results before and after the model update process to calibrate $k$ and $c_{v}$ of Top made of 303steel. (a) Temperature results and (b) mean squared error (MSE) of sensors.
TABLE IV: INITIAL AND UPDATED VALUES OF THE THERMAL PARAMETERS FOR EACH MATERIAL

\begin{tabular}{ccccccc}
\hline \multirow{2}{*}{ Material } & \multicolumn{2}{c}{ Initial values } & \multicolumn{2}{c}{ Updated values } & \multicolumn{2}{c}{ Typical values } \\
& $c_{v}$ & $k$ & $c_{v}$ & $k$ & $c_{v}$ & $k$ \\
\hline 303steel & 450.0 & 15.0 & $\mathbf{5 1 5 . 7}$ & $\mathbf{1 9 . 3}$ & 500.0 & 16.2 \\
AL6061 & 900.0 & 150.0 & $\mathbf{9 5 4 . 4}$ & $\mathbf{2 0 1 . 1}$ & 897.0 & 167.0 \\
\hline
\end{tabular}

TABLE V: INITIAL AND UPDATED VALUES OF THE THERMAL CONTACT CONDUCTANCE AT CONTACT SURFACES

\begin{tabular}{lcc}
\hline Material Pairs & $\begin{array}{c}\text { Initial values } \\
h\end{array}$ & $\begin{array}{c}\text { Updated values } \\
h\end{array}$ \\
\hline AL6061-AL6061 & 8,000 & $\mathbf{1 3 , 2 3 0}$ \\
AL6061-303steel & 1,000 & $\mathbf{2 , 1 5 8}$ \\
AL6061-Heat sink & 8,000 & $\mathbf{7 , 3 9 4}$ \\
\hline
\end{tabular}

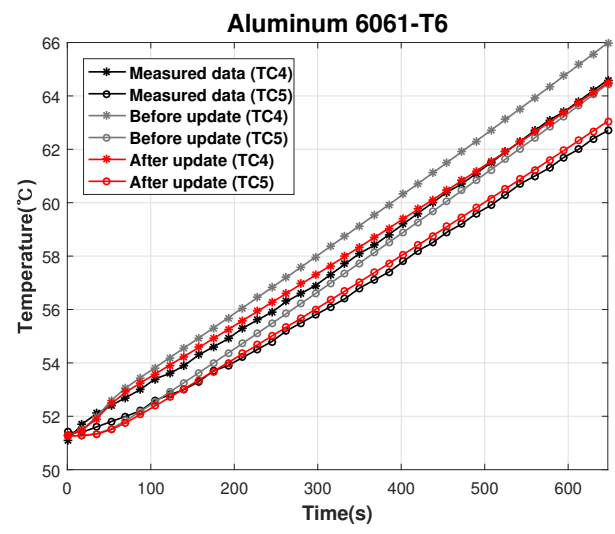

(a)

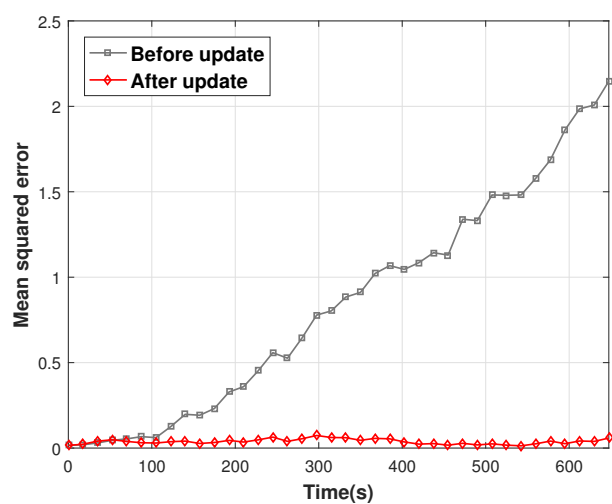

(b)

Fig. 7: Comparison of results before and after the model update process to calibrate $k$ and $c_{v}$ of Bottom made of AL6061. (a) Temperature results and (b) mean squared error (MSE) of sensors. 


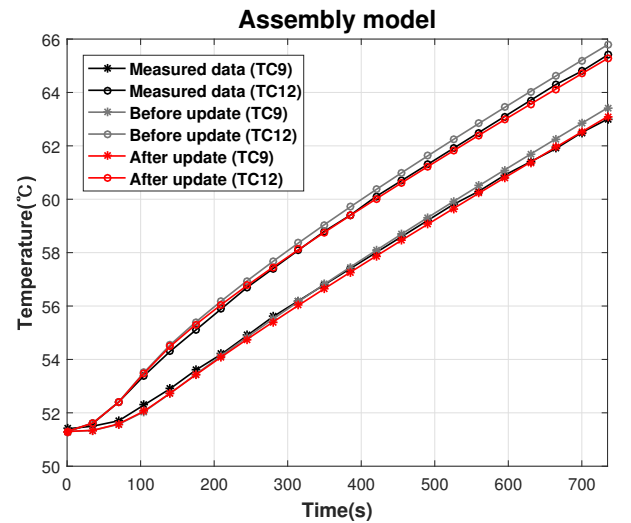

(a)

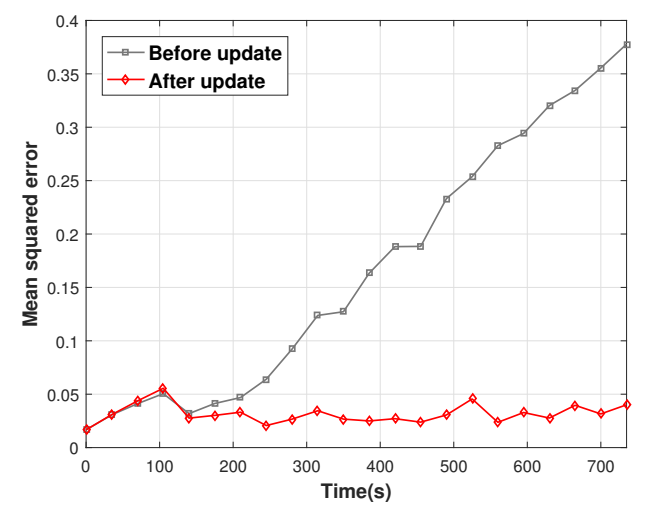

(b)

Fig. 8: Comparison of results before and after the model update process to calibrate the thermal contact conductances. (a) Temperature results and (b) mean squared error (MSE) of sensors.

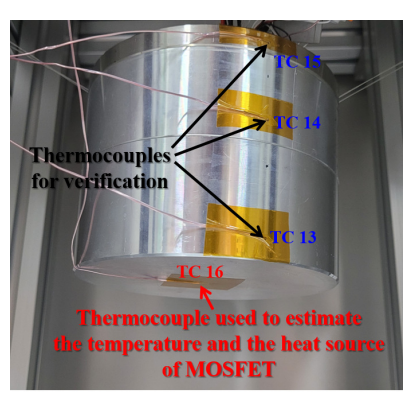

(a)

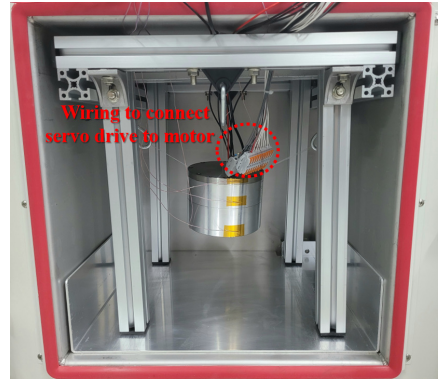

(b)
Fig. 9: A temperature sensor for virtual sensing is attached to the floor of the measurement target, and four temperature sensors are used to validate the VTS.

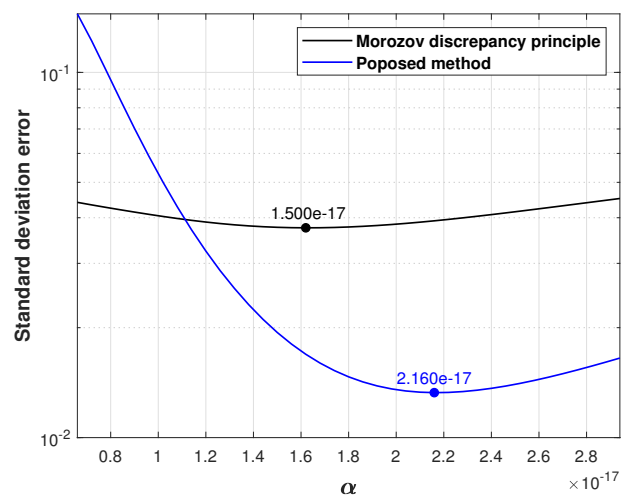

Fig. 10: Standard deviation error results of the ridge estimators when $\alpha$ is varied. The average elapsed times to estimate $\alpha$ by the Morozov and the proposed methods are 16.13 hours and 1.29 hours, respectively.

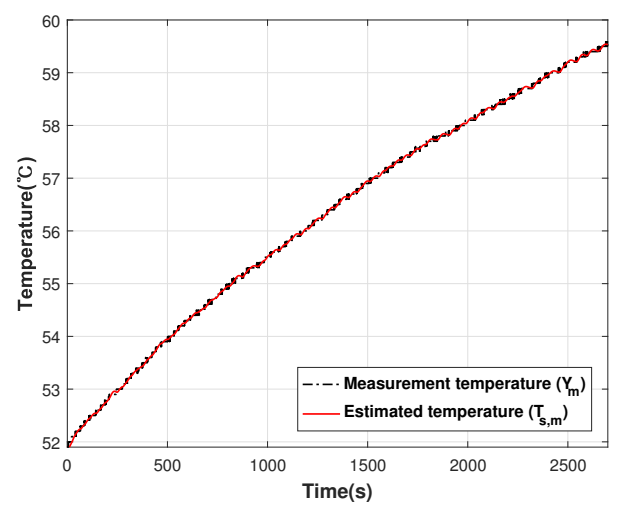

(a)

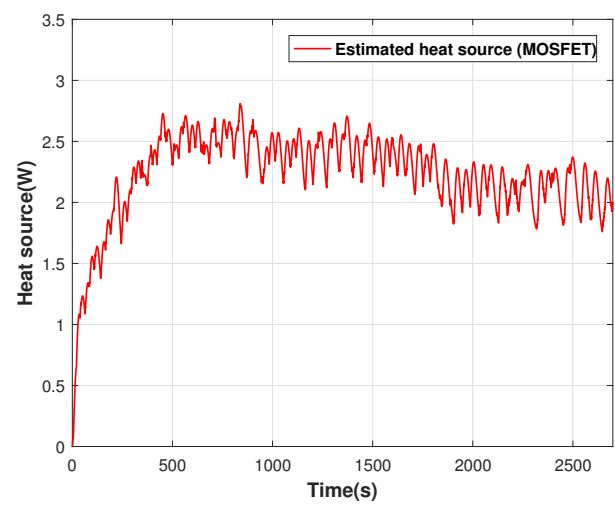

(b)

Fig. 11: Heat source of the MOSFET instrumented by a virtual thermal sensor using a T-tpye thermocouple. The regularization parameter $\alpha$ adopted for stability is $2.16 \times 10^{-17}$. 


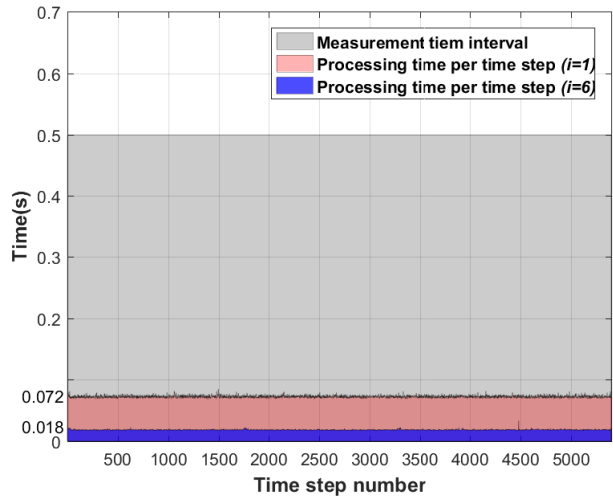

Fig. 12: Processing time per time step in the processing unit for virtual sensing. The measurement time interval of the thermocouple data logger is $0.5 \mathrm{sec}$. In the legend, $i$ denotes the number of subdomains decoupled by the Schur complement.

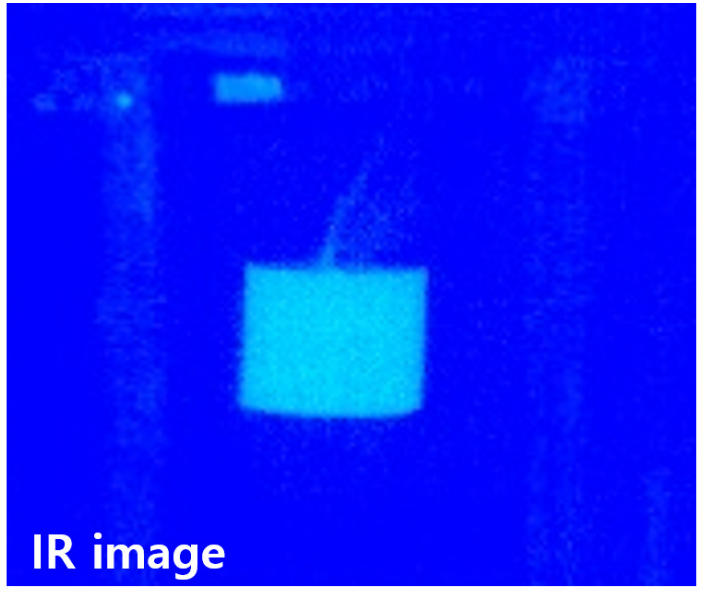

※ Operating time of motor: $40 \mathrm{~min}$

(a)

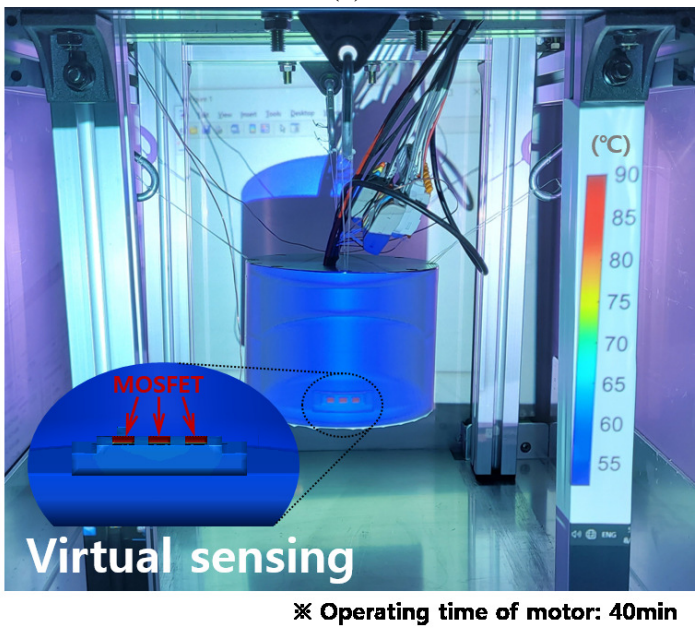

(b)

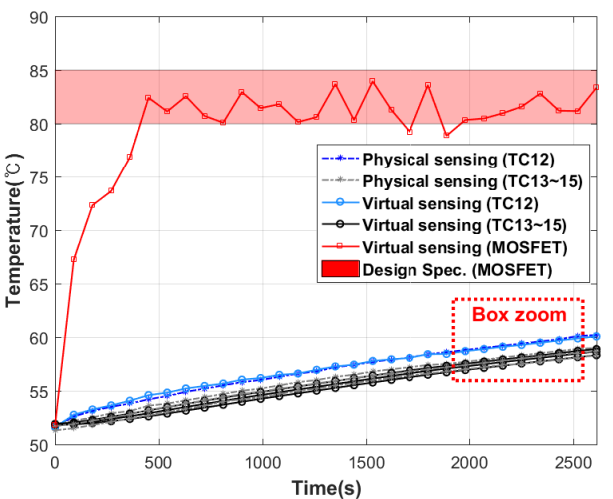

(a)

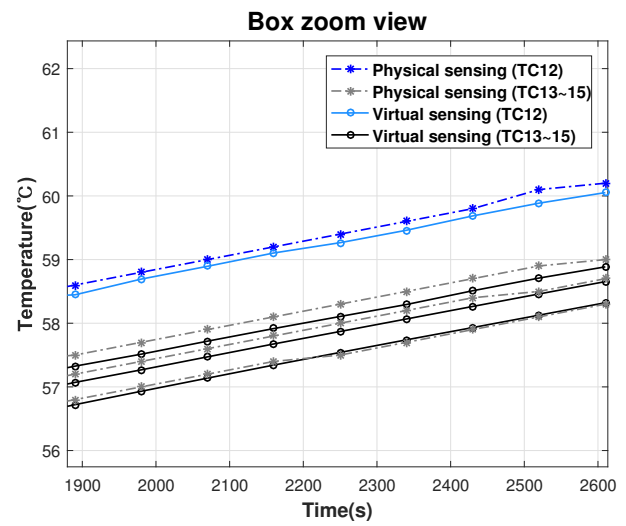

(b)

Fig. 14: Compare temperature results measured by the thermocouple and the virtual thermal sensor for validation.

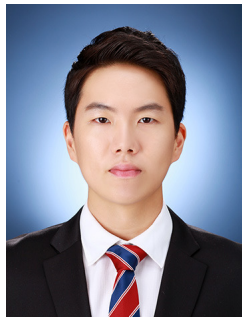

Chang-uk Ahn was born in Korea and received his B.S. degrees in Department of Mechanical Engineering from Konkuk University in 2016. He is currently a Ph.D. candidate in Department of Mechanical Engineering at Kyung Hee University. His research interests include Thermomechanical analysis, inverse heat conduction problem, and virtual thermal sensing.

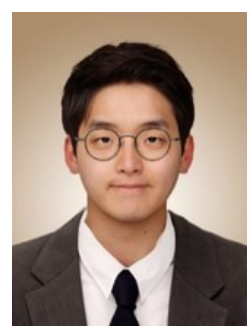

Seungin Oh was born in Korea and received his B.S. and M.S. degrees in Department of Mechanical Engineering from Kyung Hee University in 2019 and 2020, respectively. $\mathrm{He}$ is currently a Ph.D. candidate in Department of Mechanical Engineering at Kyung Hee University. His research interests include Model based digital twin, inverse force identification, virtual sensing, and vibro-acoustic interaction.

Fig. 13: Temperature distribution images taken with the IR camera and internal temperature distribution images obtained with the virtual thermal sensor. 


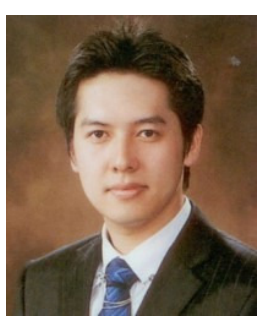

Hwi-Su Kim was born in Korea and received his B.S. and Ph.D degree in Mechanical Engineering from Korea University, in 2007 and 2014, respectively. Dr. Kim was with the School of Mechanical Engineering at Korea University. And he is currently a senior researcher at Korea Institute of Machinery and Materials (KIMM). His research interests include manipulator design, counterbalance mechanism and safe robot arms.

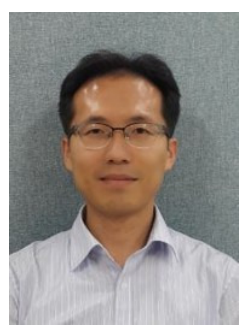

Dong II Park was born in Korea and received his B.S., M.S., and Ph.D degree in Mechanical Engineering from Korea Advanced Institute of Science and Technology (KAIST) in 2000, 2002, and 2006, respectively. He has been researching robotics and mechatronics in Korea Institute of Machinery and Materials (KIMM) since 2006. His research interests are the design and analysis of mechanical systems. He is also interested with the modeling, control and application of robot manipulators and mobile robots.

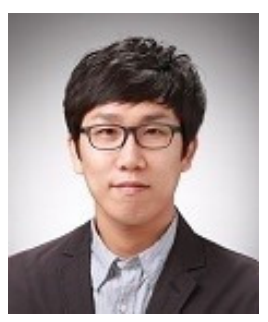

Jin-Gyun Kim was born in Korea and received his B.S and M.S degrees in Civil Engineering from Korea University in 2008 and 2010, respectively, and his Ph.D. in ocean systems engineering from Korea Advanced Institute of Science and Technology (KAIST) in 2014. He worked as a senior researcher in Korea Institute of Machinery and Materials (KIMM) from 2014 to 2017. $\mathrm{He}$ is currently an Assistant Professor of Kyung Hee University of Mechanical Engineering. His research area is computational dynamics, vibration, Multiphysics modeling and simulation. 